\title{
Lubricant flow in thin-film elastohydrodynamic contact under extreme conditions
}

\author{
P. SPERKA*, I. KRUPKA, M. HARTL \\ Faculty of Mechanical Engineering, Brno University of Technology, Technicka 2896/2, Brno 61669, Czech Republic \\ Received: 26 September 2016 / Revised: 07 November 2016/Accepted: 25 November 2016 \\ (C) The author(s) 2016. This article is published with open access at Springerlink.com
}

\begin{abstract}
To further extend knowledge about fluid film friction in elastohydrodynamic contact, it is important to examine how lubricant flows. In this paper, several film thickness results obtained by interferometry technique for different kinds of experiments were analyzed and discussed based on lubricant flow continuity. Results of two steady-state and two transient experiments are presented. Possible speed profiles that can explain observed film thickness distributions were suggested. It is shown that major part of present experiments can be explained by a single speed profile known as a plug flow. This finding is in contradiction to usual linear speed profile predicted by Reynolds equation.
\end{abstract}

Keywords: fluid film friction; speed profile; flow rate continuity; lubricant rheology; plug flow

\section{Introduction}

Elastohydrodynamic lubricant (EHL) is a regime of non-conformal contact lubrication that enables low friction and long life operation of machine elements. It is thanks to the formation of coherent lubricating film avoiding a contact between surfaces. In this case, the friction produced in a contact depends on viscous dissipation of energy in the film. Lubricant rheology undergoes a significant change inside contact under loading by pressure and shear rate.

The most lubricants change its viscosity with pressure approximately exponentially, therefore, it is surprising that EHL contact exhibits low coefficient of friction. This question has been recognized for many years [1], but it was not sufficiently answered yet. Despite, a significant progress has been made in high-speed regime during last decade, the origin of friction in low-speed, high load regime still remains a challenging problem [2]. The present explanation holds on concept of limiting shear stress suggested by Smith $[1,3]$.

\footnotetext{
* Corresponding author: P. SPERKA.
}

E-mail: sperka@fme.vutbr.cz
Generation of fluid film friction inside a contact is connected with fluid flow and through-film shear rate distribution. It defines the importance of a method allowing to obtain information about speed profile. Several kinds of known speed profiles and phenomena affecting them are summarized in Fig. 1. Essentially, domination of Counette term of Reynolds equation over Poiseuille term leads to linear speed profile (Fig. 1(a)) in highly loaded central zone of an EHL contact. In case of high sliding speed important temperature rise is generated in the contact. The highest temperature is localized inside a lubricant layer due to low thermal conductivity of a lubricant. It is connected with relative higher shear rate around the center of speed profile (Fig. 1(b)) corresponding to low local viscosity of lubricant [4-7].

Some publications reported experimental or numerical observation of shear zone localized in the center of the speed profile (Fig. 1(c)) [1, 8-12]. The shear zone represents a place with extreme shear rate and it is connected with a steep jump in speed profile. It was discussed the connection of this speed profile with failure or plastic behavior of lubricating film under shear. The consideration of plug-flow (Fig. 1(d)) to explain dimple phenomena was suggested by 
(a) Couette flow

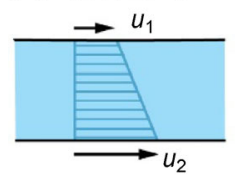

(d) Plug flow

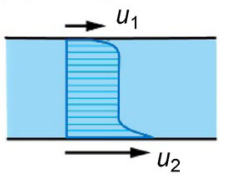

(b) Temperature rise

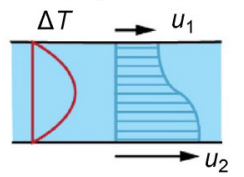

(e) Boundary slippage

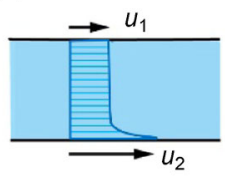

Fig. 1 Types of through-film speed profiles.

Ehret et al. [13]. In this speed profile, two shear zones are formed close to surfaces while core of lubricant is transported at the mean speed. This flow mechanism can explain transportation of perturbed film in a rolling-sliding EHL contact [14-17], moreover, it was independently observed by photobleaching technique [18]. Boundary slippage or slip on wall (Fig. 1(e)) is another phenomenon that might occur in an EHL contact affecting speed profile. It occurs when shear strength of interface between lubricant and surface is weaker than shear stress transferred by lubricant [19-22]. However, it has to be considered that boundary slippage can occur suddenly and only at one interface which is connected with modification of average lubricant speed in the speed profile. Therefore, if it happens rapid changes (increase) of film thickness can be visible [23].

The aim of this paper is to interpret film thickness results of several experiments for possible speed profile based on lubricant flow continuity. The condition of constant volume flow rate applied on film thickness results with steady-state variations gives advice on possible speed profile. Results of two steady-state and two transient experiments are presented.

\section{Background}

Reynolds equation is the most often considered in numerical solution of elastohydrodynamic lubrication problem. The equation is used in a form

$\frac{\partial}{\partial x}\left(\frac{\rho h^{3}}{12 \eta} \varphi_{x} \frac{\partial p}{\partial x}\right)+\frac{\partial}{\partial y}\left(\frac{\rho h^{3}}{12 \eta} \varphi_{y} \frac{\partial p}{\partial y}\right)-u_{\mathrm{m}} \frac{\partial(\rho h)}{\partial x}-\frac{\partial(\rho h)}{\partial t}=0$

This equation consists of so-called Poiseuille, Couette

and squeeze terms, respectively. In piezoviscous-elastic regime where pressure is high the viscosity of lubricant increases substantially. Inside central zone the viscosity reaches high value, therefore, relative importance of the Poiseuille term is negligibly small compared to the Couette term. Generally, the squeeze term is necessary to consider for transient conditions. But, if there is no change of film thickness profile over the time, the squeeze term will be zero and transient problem converts to stationary. Then, the Reynolds equation simplifies to

$$
\frac{\partial(\rho h)}{\partial x}=0
$$

where $\rho$ is density, $h$ is film thickness and $x$ is coordinate in rolling direction. By assuming no-slip boundary condition a common solution of Eq. (2) is

$$
u(z)=u_{1}+\left(u_{2}-u_{1}\right) \frac{z}{h}
$$

where $z$ is through-film coordinate, $h$ is film thickness, and $u_{1}, u_{2}$ are speeds of contact surfaces. This means that Reynolds equation gives a linear through-film speed profile inside central zone.

Essentially, mass continuity has to be maintained inside contact. If density variations are considered negligible small, the mass continuity equation transforms to volume continuity equation. For laminar flow in entrainment direction it expresses conservation of volume flow rate. Volume flow rate in entrainment direction at any point inside contact can be calculated by integration of lubricant speed through film thickness.

$$
Q=\int_{z} u(z) \mathrm{d} z=h \cdot \bar{u}
$$

It is equal to film thickness multiplied by average speed of lubricant. In a speed profile, volume flow rate is proportional to its area.

$$
Q_{x}=h_{x} \cdot \bar{u}_{x}=\text { const. }
$$

If no side flow, no through-film flow and incompressible lubricant is assumed and film thickness profile is at steady state, volume flow rate has to be constant all over the contact ( $x$ coordinate).

Therefore, any variation in film thickness has to be 
connected with change of the average speed of lubricant. If no slip at the interface between surface and lubricant is expected, the speed of lubricant at the interface is equal to the speed of surface. Then, the speed profile shape is changed which corresponds to variations of average speed of lubricant. This enables interpretation of steady-state film thickness results for possible speed profiles. However, it cannot provide a particular speed profile shape with no ambiguity. Rather this approach can advise which speed profile from series of rational options is able to explain the observed in-contact film variations based on flow continuity.

The assumption of no side flow is generally well fulfilled in central high-pressure zone because it is caused by pressure driven flow (Poiseuille flow) which is small relative to other flows. Moreover, often centreline profile of point contact is analysed where pressure gradients in transverse axis are zero. At some cases the assumption of no through-film flow is questionable. Then, an alternative speed profile configuration with non-zero through-film flow can be considered as it is done in case of pure opposite sliding. There is a possibility of through-film flow quantification by using conservation of total volume flow rate.

\section{Methods and material}

Film thickness data presented in this paper were measured using colorimetric interferometry [24, 25] and a ball-on-disc tribometer. This apparatus enables simulation of lubricated point contact between a steel ball and a glass disc. Both surfaces are independently driven by servomotors and loaded against each other by dead weight through a lever system. The device was extended for possibility of ball axis rotation to allow study conditions of arbitrary velocity directions [26]. The current experimental rig setup has a high power xenon continues lamp and a high speed CMOS camera for observation of transient phenomena. The colorimetric interferometry method measures film thickness by incoherent white light interference occurring between two beams reflected from bottom disc surface and ball surface. The reflectance of disc bottom surface is ensured by thin layer of chromium without any spacer layer. Calibration is based on monochromatic and chromatic images of the same static contact. It uses monochromatic interferogram to obtain real geometry of the clearance and assign film thickness to particular color found in chromatic interferogram to made calibration curves. Robust and efficient implementation of calibration curves allows rapid film thickness evaluation of measured interferograms by searching for minimum difference between colour from interferogram and calibration curves. Full and detail description of the method and evaluation technique can be found in Refs. [24, 25].

Combination of glass disc and bearing steel ball (100Cr6) of $25.4 \mathrm{~mm}$ diameter giving reduced modulus of elasticity $123.8 \mathrm{GPa}$ was used in this study. One experiment was conducted with sapphire disc which gives against steel ball reduced modulus of elasticity $314 \mathrm{GPa}$. All experiments instead of one were conducted with smooth surface. Transverse ridge produced by sputtering of chromium on smooth steel ball was used in one experiment. The ridge was $60 \mu \mathrm{m}$ wide and $250 \mathrm{~nm}$ high. This paper includes experiments for three mineral base oils SR150, SR600 and N500 having ambient viscosities $0.057,0.23$ and $0.32 \mathrm{~Pa} \cdot \mathrm{s}$ respectively and pressure-viscosity coefficients of 22.5, 24 and $31 \mathrm{GPa}^{-1}$ respectively. These values correspond to temperature at which the experiments were carried out. The range of temperatures for all experiments is $26 \pm 2{ }^{\circ} \mathrm{C}$.

\section{Results and discussion}

Figure 2 shows interferogram and corresponding centerline film thickness profiles of an EHL contact under pure rolling and rolling-sliding conditions. Measurements are for steel-glass contact, SR150 oil, $0.55 \mathrm{GPa}$ Hertzian pressure and mean speed $0.5 \mathrm{~m} / \mathrm{s}$. Slide-to-roll ratio in Fig. 2(b) is $100 \%$ which means that glass disc is faster than ball. In the figure it can be seen that the profile is practically the same in both cases, only, complete profile is slightly shifted down to smaller film thickness presumably due to thermal effect of sliding in the contact inlet. It was already well recognized that the shape of film thickness profile inside contact is given by density change. It means that mass continuity is maintained, and if density change is neglected the volume flow rate is 

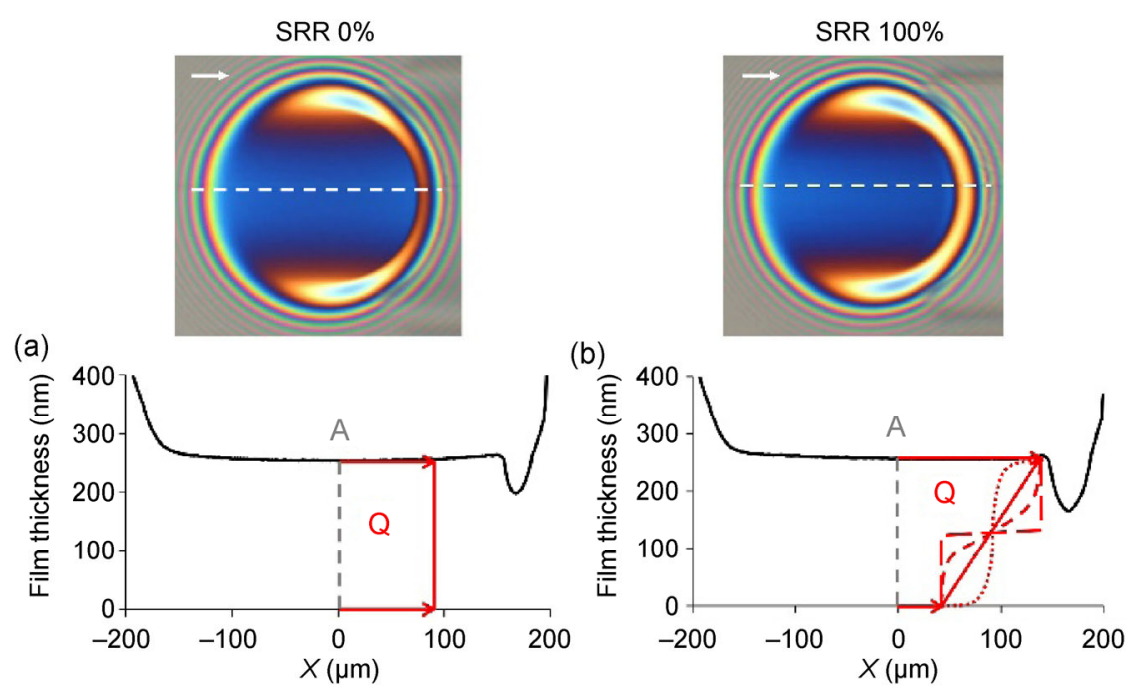

Fig. 2 Interferograms and centerline film thickness profiles with illustrated speed profile under (a) pure rolling; (b) rolling-sliding.

constant in both cases. The speed profile in Fig. 2(a) is evident, thanks to high viscosity Poiseuille flow which is insignificant to Couette flow. The speed profile in Fig. 2(b) is according isothermal theory and Reynolds equation presented in Section 2 linear, and if thermal effects are considered temperature rise in the film will make speed profile non-linear. The shape of non-linearity depends on thermal properties of contact bodies [7], namely heat diffusivity. For steel-glass contact the highest temperature will be localized close to glass disc. Nevertheless, the other two options of speed profile, namely plug-flow and central slip, cannot be excluded from consideration in Fig. 2(b).

Figure 3 presents interferogram and film thickness profiles of conditions where ball and disc surface have arbitrary directions shown by arrows. In this case entraining velocity coincides with $x$ axis and sliding velocity with $y$ axis. Moreover, the velocity components of both surfaces in $x$ direction are the same, therefore, there is a pure rolling in $x$ direction. The ratio between sliding and entraining velocities magnitudes (SRR) is equal to $483 \%$. The result was obtained for glass-steel contact with mineral base oil N500, Hertzian pressure $0.7 \mathrm{GPa}$ and entrainment speed $0.16 \mathrm{~m} / \mathrm{s}$. The flow continuity should be maintained in $2 \mathrm{D}$ space, naturally because there is a pure rolling in $x$ direction, so there is only Couette flow in $y$ direction. The plot of film thickness in $y$ direction exhibits a wedge shape. The wedge shaped film is generated initially at the inlet. It is expected that it is due to different thermal properties of contact bodies and connected unsymmetrical temperature rises. The point of this paper is to focus on in-contact film variations and possible connection to a lubricant flow.

The inclination of the wedge is increasing with increasing distance in $x$ (entraining) direction. Surfaces are making pure opposite sliding in $y$ direction and pure rolling in $x$ direction. Therefore, film profile modification in $y$ direction is connected with lubricant flow under pure opposite sliding. One possible configuration of speed profiles at two points A and B is shown in Fig. 3(a). Under assumption of laminar flow, a linear speed profile should be connected with larger volume flow rate directed to left $Q_{\mathrm{L} 2}$ in point $\mathrm{B}$ than that directed to right $Q_{\mathrm{R} 1}$ in point $\mathrm{A}$. As a consequence, the wedge shape inclination should decrease with increasing distance in $x$. However, it is not the case, therefore, $Q_{\mathrm{R} 1}$ and $Q_{\mathrm{L} 1}$ have to be the same or slightly larger than $Q_{\mathrm{R} 2}$ and $Q_{\mathrm{L} 1}$. This can take place in two principal ways. One is linear speed profile in point $A$ and speed profile in point $B$ has to be non-linear as it is shown by blue dash-dot line (Fig. 3(a)). Other is a linear speed profile in point B and green dash line profile in point A (Fig. 3(b)). Alternatively, there can be the combination of two non-linear speed profiles with condition of the same volume flow rates to individual sides in points $\mathrm{A}$ and B.

Another interesting case represents condition of 

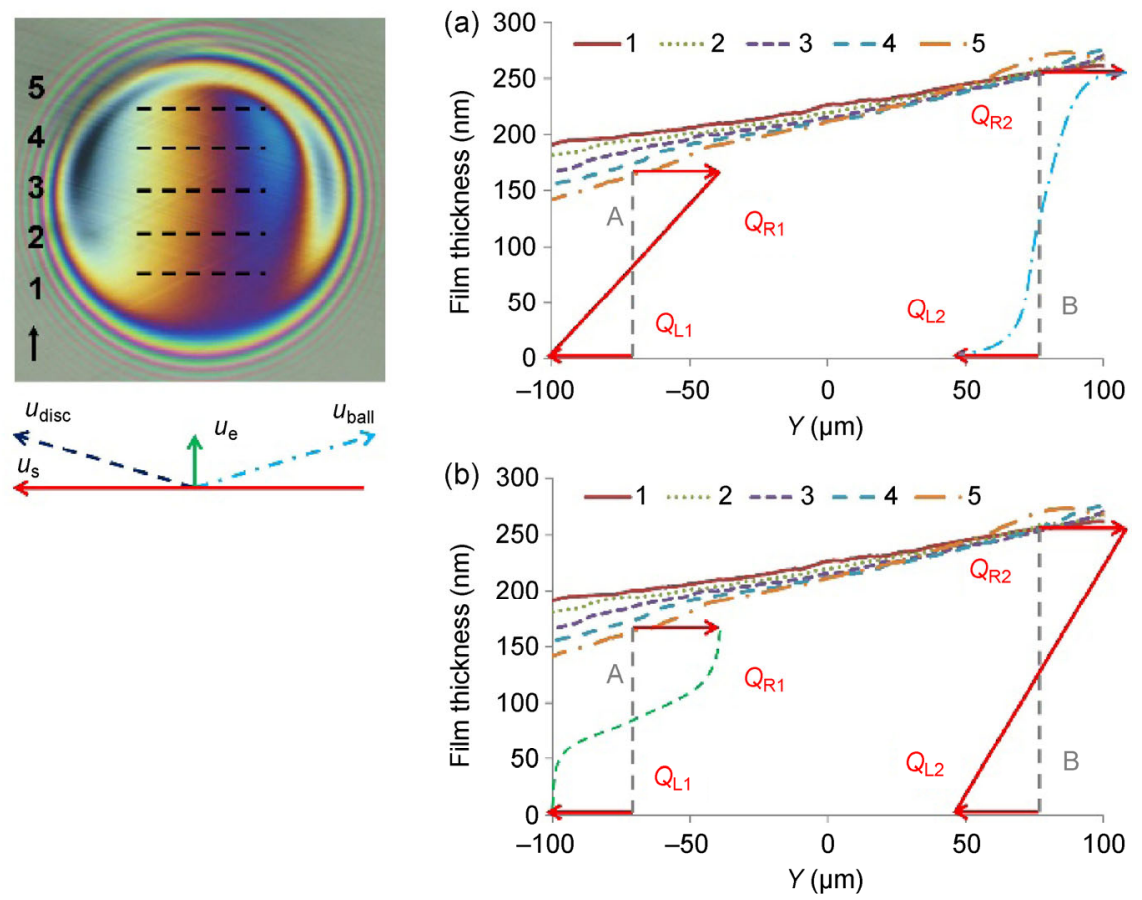

Fig. 3 Interferogram of an EHL contact under arbitrary velocity directions and film thickness profile perpendicular to entraining velocity.

pure opposite sliding (also called zero entrainment velocity condition). In this case, a deep symmetrical dimple of thick film surrounded by thin film rim is formed in central zone as it is shown in Fig. 4. This result was obtained for sapphire-steel contact, mineral base oil N500, Hertzian pressure 1.0 GPa and sliding speed $1 \mathrm{~m} / \mathrm{s}$. Here, SRR is equal to $\infty$. It is important to note that the ratio between central film thickness $h_{\mathrm{c}}$ and film thickness in thin film rim $h_{1}$ is roughly equal to 8 and this phenomenon is steady state. Obviously, volume flow rate has to be maintained, therefore, two principal options of lubricant flow can be sketched. One is expecting existence of through-film flow and the other not. Both options are schematically shown in Fig. 5.

In Fig. 5(a) speed profiles are shown in two points $C$ and D. For simplicity a linear speed profile is considered in point $C$ despite non-linear profile is more realistic there due to thermal effects. Speed profile in point $\mathrm{D}$ has a shape given by significant temperature rise inside film connected with decrease of viscosity and high shear rate. Derivation of speed profile at any point in through-film coordinate gives local shear rate. Even from the area of speed profiles it is evident that volume flow rate directed to the right $Q_{\mathrm{R} 1}$ in point $\mathrm{C}$ is much smaller than volume flow rate to the right $Q_{\mathrm{R} 2}$ in point $\mathrm{D}$. Therefore, secondary through-film flow, shown in cross-section E, should exist. As a result, the lubricant might rotate in a contact. The other option is shown in Fig. 5(b). With the same expected speed profile in point $C$ and no through-film flow, the speed profile in point $D$ has to have a shape giving the same volume flow rate $Q_{\mathrm{R} 2}$ and $Q_{\mathrm{R} 1}$. Since the film thickness in point $\mathrm{D}$ is much larger than that in point $C$, the speed profile in point $\mathrm{D}$ contains non-zero speeds close to the surfaces and the main part of lubricant is steady on position (practically zero speed) inside contact.

The dimple phenomenon can be explained by thermal viscosity wedge. It expects viscosity gradients in through-film direction which is caused by difference in duration of lubricant heating-up that flows to the left and to the right side, except in the mid-point. This fact will produce non-zero average flow everywhere except in mid-point. Moreover, due to high sliding speed significant temperature rise will occur. It will decrease lubricant viscosity and Poiseuille flow can no more be ignored. Therefore, it seems that the option with through-film flow, shown in Fig. 5(a), is more probable. It means the lubricant rotate inside the 

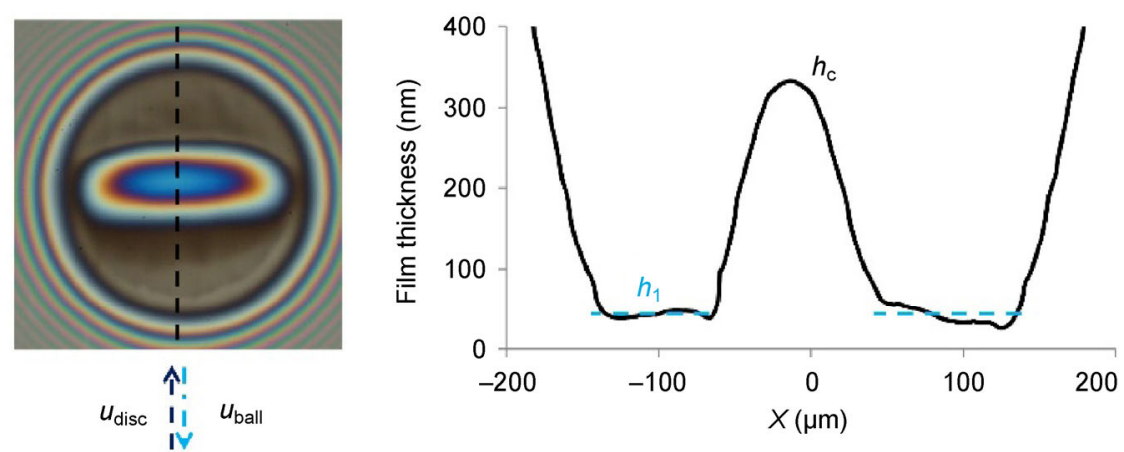

Fig. 4 Interferogram and centerline film thickness profile of an EHL contact under pure opposite sliding.
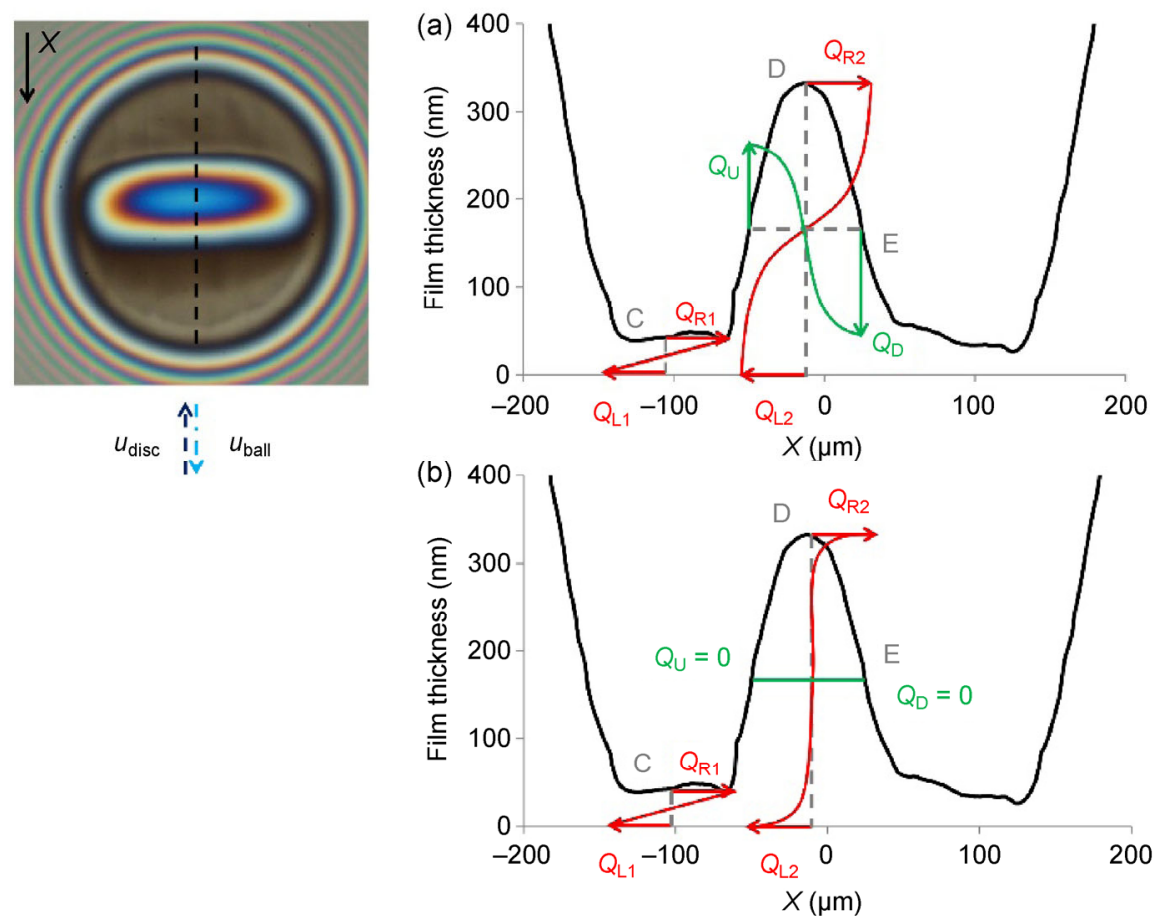

Fig. 5 Illustration of speed profiles in the contact under pure opposite sliding (a) with and (b) without through-film lubricant flow.

contact which increases time for lubricant heating-up.

For option shown in Fig. 5(a), there is a possibility to quantify through-film speed directly by applying volume continuity equation. Because dimensions in profile have different scales in $x$ and $y$ directions, estimated maximum through-film speed to explain dimple profile is $0.4 \%$ of speed of a surface.

Another group of experiments where lubricant flow will be discussed is transient conditions. Two kinds of experiments are presented in this paper. The first is experiment with smooth surface under transient rolling-sliding conditions. The time-progress of ball and disc speeds is shown in Fig. 6. Initially speed of ball and disc are steady at SRR equal to -1 (ball faster than disc), after, the ball is stopped for small period of time (typically $5 \mathrm{~ms}$ ). During the stopping phase the lubricant inside contact is entrapped while the film thickness decreases on the edges and forms a rim shape constriction. Subsequently, the speed of ball is rapidly increased to initial value, so the SRR = -1 is maintained. At this time the rim constriction is entrained into the contact under rolling-sliding conditions. Evaluation of film thickness profile changes of this constriction propagation through contact area is a key point for this observation. Figure 7 shows time evolution of the propagation on images with 


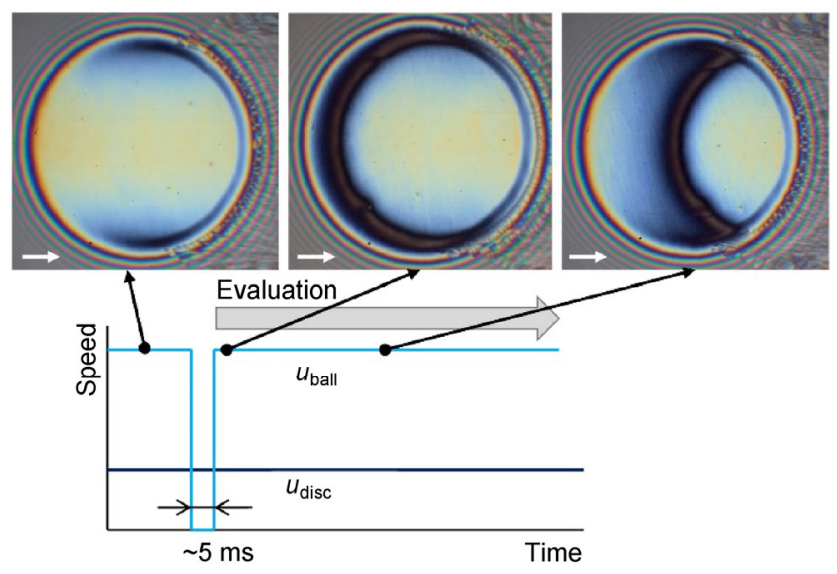

Fig. 6 Scheme of contact surfaces speeds evolution.

interferogram and corresponding centerline profiles in entrainment direction. It was obtained for mineral base oil SR600, Hertzian pressure 0.63 GPa and mean speed of $0.06 \mathrm{~m} / \mathrm{s}$.

The constriction shown in Fig. 7 propagates through the contact at mean speed. Therefore, to observe changes in the profile during passage it is necessary to change the frame of reference. Naturally, the observation is done by fixed position camera, and it gives Eulerian frame of reference fixed outside of the contact. It is necessary to change this frame to the Lagrangian frame of reference fixed to average speed of lubricant which, in this case, is the mean speed of surfaces. This conversion is done by plotting data to modified $x$-axis coordinate defined as

$$
x^{\prime}=x-u_{\mathrm{e}} \cdot t
$$

where $u_{\mathrm{e}}$ is the mean speed of surfaces and $t$ is time of the frame.

Figure 8 presents results from Fig. 7 plotted against modified $x^{\prime}$ coordinate. On the left side of the figure there is a contact inlet. Numbers shown next to the lines depict time in ms corresponding to profile. From the figure it is evident that the shape of film thickness profile around the constriction does not change. Now, to discuss possible speed profiles two arbitrary points $\mathrm{A}$ and $\mathrm{B}$ were considered. Due to frame of reference conversion, a speed profile is drawn relative to the mean speed. Thus, upper surface has higher speed than the mean speed which corresponds to speed of ball and speed of bottom surface matches to relative speed of disc. No through-film flow and no side flow is assumed. Afterwards, because there is no significant change of film thickness (it is in steady state against Lagrangian frame of reference) the volume flow rate $Q_{\mathrm{R} 1}$ must be equal to $Q_{\mathrm{R} 2}$ and the volume flow rate $Q_{\mathrm{L} 1}$ equal to $Q_{\mathrm{L} 2}$. Linear speed profile expected in point B leads to highly non-linear speed profile in point A. Alternatively, to achieve linear speed profile in point $A$, the speed profile in point $B$ has to include speeds roughly four times greater than speed of ball or disc. Theoretically, large Poiseuille flow can explain such a speed profile, but it is not realistic due to large lubricant viscosity. Therefore, the situation illustrated in Fig. 8 is more probable. The experiment shown in Figs. 6-8 is transient, therefore, generally the squeeze term of Reynolds equation (Eq. (1)) should be considered as well. However, because the plot in Fig. 8 shows no change of film profile over time, squeeze term is not important and the problem is converted to stationary.

The second transient experiment represents observation of film thickness variations induced by transverse ridge under steady rolling-sliding conditions.

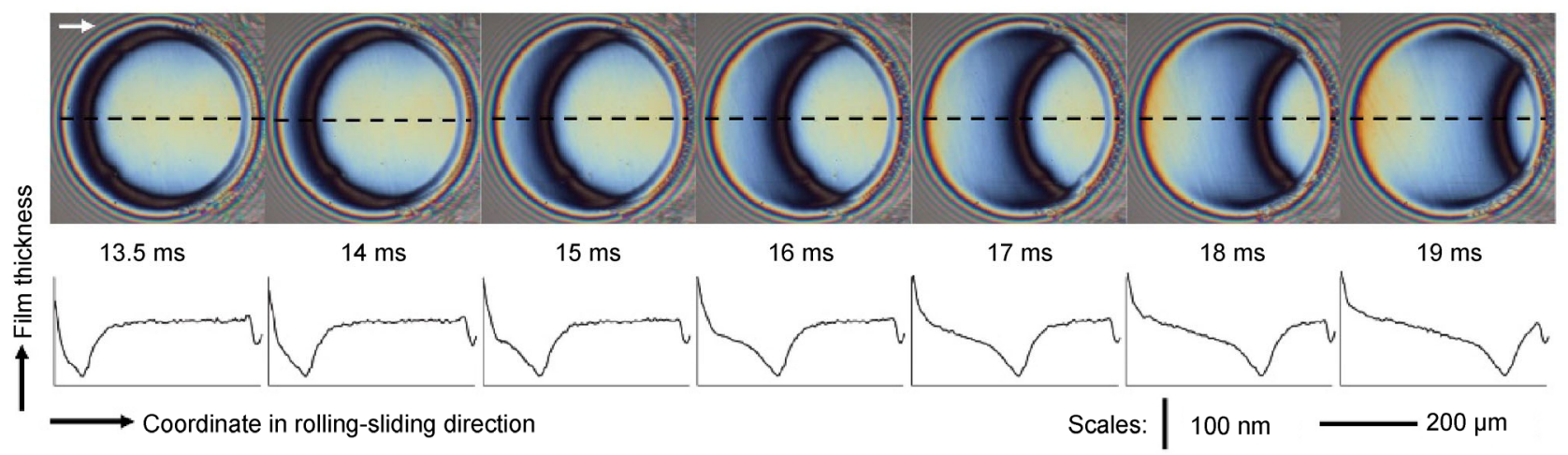

Fig. 7 Evolution of film thickness during propagation of constriction. 
The same kind of experiment has been already published by present authors [16]. It is included to compare results with other results. Time-evolution of film thickness variations are shown in Fig. 9 on interferograms and centreline profiles. Only six frames from series of twelve and the parts of profile important for further analysis are shown in Fig. 9. This measurement was conducted with mineral base oil SR600, Hertzian pressure $0.63 \mathrm{GPa}$, mean speed of $0.06 \mathrm{~m} / \mathrm{s}$ and SRR 1 (disc faster than ball). The aim is to observe propagation of film thickness variations which moves at the mean speed across the contact. Therefore, frame of reference was transformed in the same way as in the first experiment under transient conditions. The centreline profiles are plotted against modified $x^{\prime}$ coordinate in Fig. 10. In Fig. 10(a), there are shown whole profiles including inlet and outlet during disturbed film passage across whole contact. In Fig. 10(b), there are shown only parts of profiles inside central zone.

From Fig. 10(b), it is evident that film thickness variations do not change significantly throughout the contact passage. With assumption of zero through-film flow and zero side flow in central zone, a volume flow rate in entrainment direction within whole evaluated area in Fig. 10(b) has to be the same. Two points A and B were selected and speeds are schematically drawn relative to the mean speed of surfaces. If simply linear profile is considered in point $A$, the speed profile in point $B$ has to be heavily non-linear, otherwise, the volume flow rate $Q_{\mathrm{R} 1}$ is not equal to $Q_{\mathrm{R} 2}$ and flow continuity is not fulfilled. This experiment leads to similar situation as the first transient experiment, with exception that points have switched positions. Also in this case, generally, squeeze term in Reynolds equation should be considered, but from the same reason as in previous transient experiment an explanation based on stationary equation is valid.

\section{Possibility of global speed profile}

By analysis of presented results based on lubricant continuity it was showed that major part of results can be explained by similar speed profile. In cases of

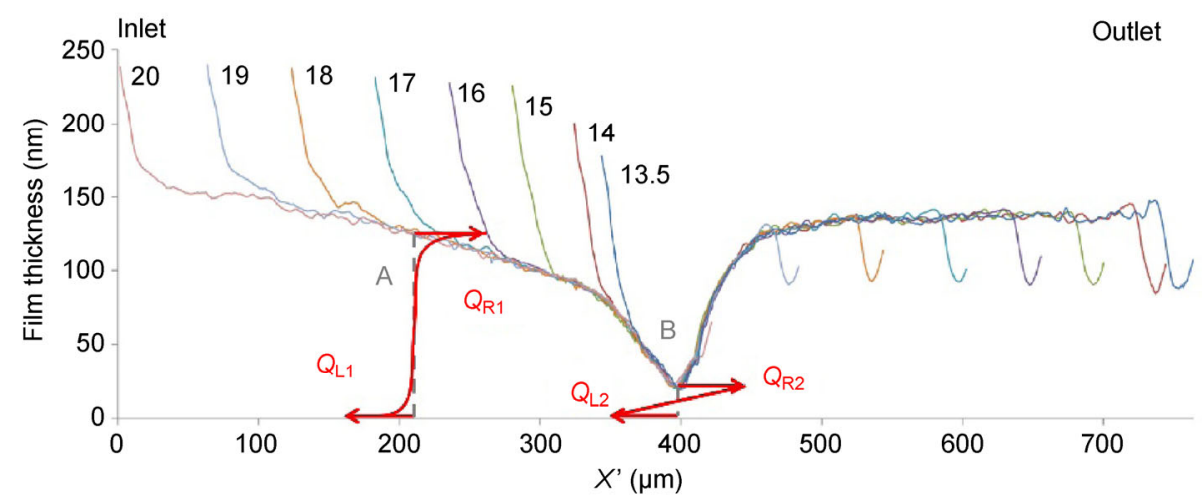

Fig. 8 Centerline film thickness profiles plotted against modified $x$ ' coordinate.

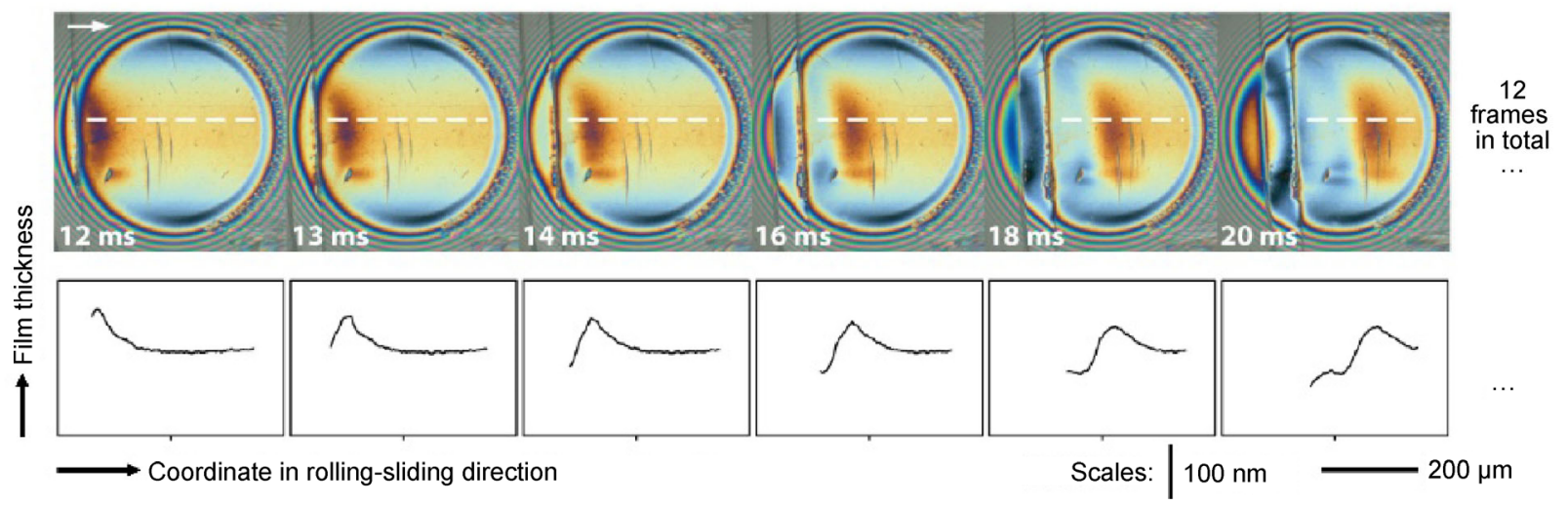

Fig. 9 Film thickness variations associated with transverse ridge under steady rolling-sliding conditions. 

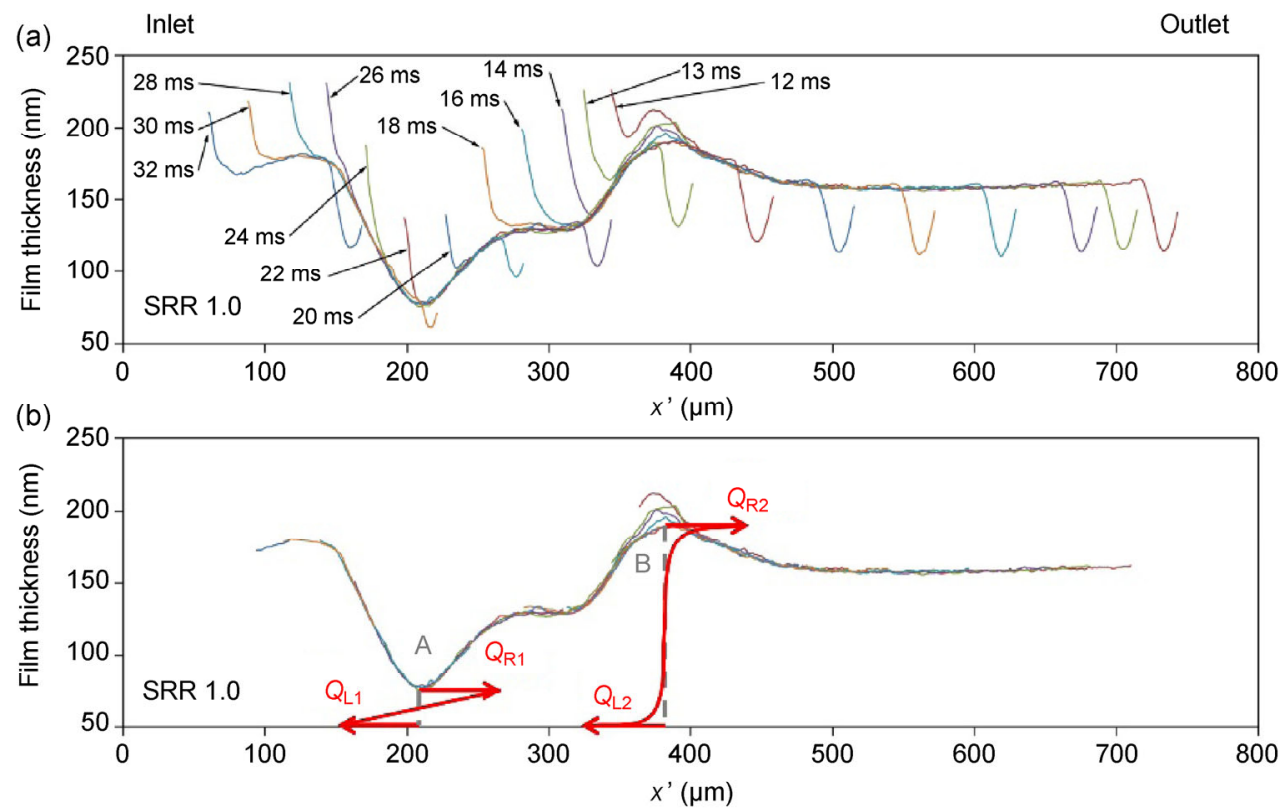

Fig. 10 Centerline film thickness profiles plotted against modified $x$ ' coordinate: (a) complete profiles; (b) parts of profiles in central zone.

Figs. 3(a), 8 and 9(b) speed profiles correspond to type of flow called plug-flow (Fig. 11). In this speed profile two shear zones are localized close to the surface, but not necessarily at the interface between surface and lubricant. Central, main part of lubricant has small shear rate inside- the plug which often moves at mean speed. At the same time, this speed profile is consistent with a film thickness profile known under simple rolling-sliding conditions (Fig. 2(b)). The speed profile was initially suggested for EHL by Ehret et al. [13] to explain dimple phenomenon. The mechanism of formation to the best authors knowledge has not been discovered yet. Some theories were already discussed elsewhere [16-18]. It seems that perspective approaches to study the phenomena are molecular dynamic simulation [11, 12] and solution of NavierStokes full system depending whether the solution is

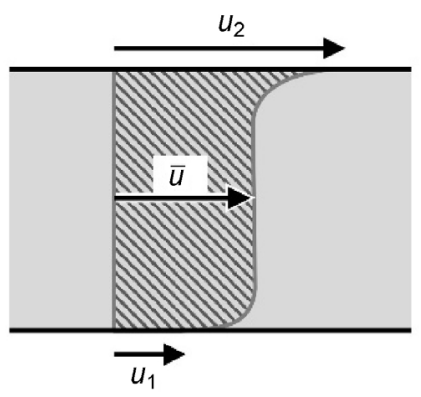

Shear zone

Plug

Shear zone

Fig. 11 Scheme of plug flow. beyond or within continuum approach. In the latter case, the same speed profile was obtained by solution of Navier-Stokes equations for fluid with pressure dependent viscosity and simple Couette flow [27, 28].

\section{Conclusions}

To further extend knowledge about fluid film friction of EHL contact, it is important to examine how lubricant flows inside the contact. In this paper, several film thickness results obtained for different kinds of conditions were analyzed and discussed based on lubricant flow continuity. Possible speed profiles which can explain observed film thickness data were suggested. It was found that major part of experiments can be explained by a single speed profile known as a plug-flow. This finding is in contradiction to usual linear speed profile predicted by Reynolds equation. Moreover, some experiments were carried out at high sliding speeds introducing a significant temperature rise into a lubricant film. Therefore, the point of the paper is not to give final conclusion rather introduce a way of looking on currently available film thickness measurements. Ultimately, the understanding of lubricant flow inside contact may give answer to fundamental question about the origin of limiting shear stress found in traction experiments. 


\section{Acknowledgement}

The research leading to these results has received funding from the Ministry of Education, Youth and Sports of Czech Republic under the National Sustainability Program I (Project LO1202).

Open Access: The articles published in this journal are distributed under the terms of the Creative Commons Attribution 4.0 International License (http:// creativecommons.org/licenses/by/4.0/), which permits unrestricted use, distribution, and reproduction in any medium, provided you give appropriate credit to the original author(s) and the source, provide a link to the Creative Commons license, and indicate if changes were made.

\section{References}

[1] Smith F W. Lubricant behaviour in concentrated contact systems-The Castor oil-steel system. Wear 2(4): 250-263 (1959)

[2] Habchi W, Bair S, Vergne P. On friction regimes in quantitative elastohydrodynamics. Tribol Int 58: 107-117 (2013)

[3] Smith F W. Lubricant behavior in concentrated contactSome rheological problems. ASLE Trans 3: 18-25 (1960)

[4] Qu S, Yang P, Guo F. Theoretical investigation on the dimple occurrence in the thermal EHL of simple sliding steel-glass circular contacts. Tribol Int 33(1): 59-65 (2000)

[5] Guo F, Yang P, Wong P L. On the thermal elastohydrodynamic lubrication in opposite sliding circular contacts. Tribol Int 34(7): 443-452 (2001)

[6] Yagi K, Kyogoku K, Nakahara T. Relationship between temperature distribution in EHL film and dimple formation. Trans ASME J Tribol 127(3): 658-665 (2004)

[7] Kaneta M, Cui J, Yang P, Krupka I, Hartl M. Influence of thermal conductivity of contact bodies on perturbed film caused by a ridge and groove in point EHL contacts. Tribol Int 100: 84-98 (2016)

[8] Bair S, Winer W O, Distin K W. Experimental investigations into shear localization in an operating EHD contact. In Tribology Series, Proc. 19th Leeds-Lyon Symposium on Tribology, Leeds, UK, 1993: 383-388.

[9] Bair S, Qureshi F, Khonsari M. Adiabatic shear localization in a liquid lubricant under pressure. J Tribol Trans ASME 116: 705-708 (1994)
[10] Bair S, McCabe C. A study of mechanical shear bands in liquids at high pressure. Tribol Int 37: 783-789 (2004)

[11] Heyes D M, Smith E R, Dini D, Spikes H A, Zaki T A. Pressure dependence of confined liquid behavior subjected to boundary-driven shear. J Chem Phys 136(13): 134705 (2012)

[12] Gattinoni C, Heyes D M, Lorenz C D, Dini D. Traction and nonequilibrium phase behavior of confined sheared liquids at high pressure. Phys Rev E 88(5): 052406 (2013)

[13] Ehret P, Dowson D, Taylor C M. On lubricant transport conditions in elastohydrodynamic conjunctions. Proc $R$ Soc Lond A Math Phys Eng Sci 454: 763-787 (1998)

[14] Kaneta M, Nishikawa H, Kameishi K. Observation of wall slip in elastohydrodynamic lubrication. J Tribol Trans ASME 112: 447-452 (1990)

[15] Kaneta M. For the establishment of a new EHL theory. In Tribology Series, Proc. 25th Leeds-Lyon Symposium on Tribology, Lyon, France, 1999: 25-36.

[16] Šperka P, Křupka I. Hartl M. Evidence of plug flow in rolling-sliding elastohydrodynamic contact. Tribol Lett 54(2): 151-160 (2014)

[17] Sperka P, Krupka I, Hartl M. Transition from plug-flow to linear speed profile near a dent in a rolling-sliding EHL contact. Tribol Int 100: 344-350 (2016)

[18] Ponjavic A, Mare L, Wong J S. Effect of pressure on the flow behavior of polybutene. J Polym Sci Part B: Polym Phys 52: 708-715 (2014)

[19] Guo F, Wong P L, Geng M, Kaneta M. Occurrence of wall slip in elastohydrodynamic lubrication contacts. Tribol Lett 34: 103-111 (2009)

[20] Guo F, Li X M, Wong P L. A novel approach to measure slip-length of thin lubricant films under high pressures. Tribol Int 46(1): 22-29 (2012)

[21] Wong P L, Li X M, Guo F. Evidence of lubricant slip on steel surface in EHL contact. Tribol Int 61:116-119 (2013)

[22] Li X M, Guo F, Wong P L. Shear rate and pressure effects on boundary slippage in highly stressed contacts. Tribol Int 59: 147-153 (2013)

[23] Yagi K, Sugimura J, Vergne P. Rheological response of fatty alcohols in sliding elastohydrodynamic contacts. Tribol Int 49: 58-66 (2012)

[24] Hartl M, Krupka I, Liska M. Differential colorimetry: Tool for evaluation of chromatic interference patterns. Optic Eng 36: 2384-2391 (1997)

[25] Hartl M, Krupka I, Poliscuk R, Liska M. An automatic system for real-time evaluation of EHD film thickness and shape based on the colorimetric interferometry. Tribol Trans 42: 303-309 (1997) 
[26] Omasta M, Křupka I, Hartl M. Effect of surface velocity directions on elastohydrodynamic film shape. Tribol Trans 56(2): 301-309 (2013)

[27] Hron J, Málek J, Rajagopal K R. Simple flows of fluids with pressure-dependent viscosities. Proc $R$ Soc Lond $A$

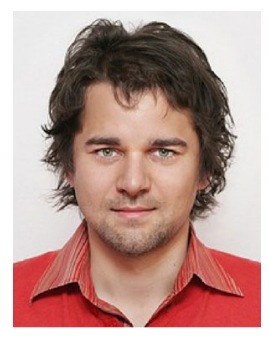

Petr SPERKA. He received his M.S. and Ph.D. degrees in mechanical engineering from Brno University of Technology, Czech Republic, in 2007 and 2011, respectively. His current position is an assistant professor and head of Elastohy-

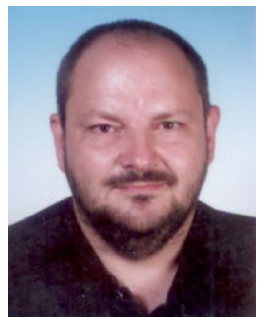

Ivan KRUPKA. He received his M.S. and Ph.D. degrees in mechanical engineering from Brno University of Technology, Czech Republic, in 1990 and 1997, respectively. His current position is a professor and

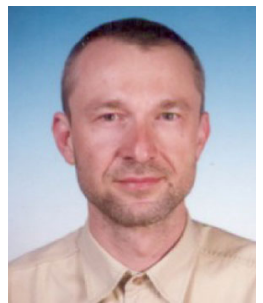

Martin HARTL. He received his M.S. and Ph.D. degrees in mechanical engineering from Brno University of Technology, Czech Republic, in 1990 and 1997, respectively. His current position is a professor and
Math Phys Eng Sci 457: 1603-1622 (2001)

[28] Hron J, Málek J, Průša V, Rajagopal K R. Further remarks on simple flows of fluids with pressure-dependent viscosities. Nonlinear Anal: Real 12: 394-402 (2011)

drodynamic Lubrication Section at Tribology Group, Institute of Machine and Industrial Design, Faculty of Mechanical Engineering, Brno University of Technology. His research areas cover the boundary, mixed and elastohydrodynamic lubrication, roughness effect, and lubricant rheology.

head of Tribology Group, Institute of Machine and Industrial Design, Faculty of Mechanical Engineering, Brno University of Technology. His research areas cover the mixed and elastohydrodynamic lubrication, surface texturing effect, and lubricant rheology.

head of Institute of Machine and Industrial Design, Faculty of Mechanical Engineering, Brno University of Technology. His research areas cover the boundary, mixed and elastohydrodynamic lubrication, roughness effect, and biotribology. 